\title{
Holomorphic Automorphisms of Danielewski Surfaces II: Structure of the Overshear Group
}

\author{
Rafael B. Andrist • Frank Kutzschebauch • \\ Andreas Lind
}

Received: 2 May 2013 / Published online: 13 June 2014

(C) Mathematica Josephina, Inc. 2014

\begin{abstract}
We apply Nevanlinna theory for algebraic varieties to Danielewski surfaces and investigate their group of holomorphic automorphisms. Our main result states that the overshear group, which is known to be dense in the identity component of the holomorphic automorphism group, is a free product.
\end{abstract}

Keywords Danielewski surface · Overshear group · Nevanlinna theory · Holomorphic automorphism group

Mathematics Subject Classification Primary 32M17 - Secondary 32A22

\section{Introduction}

The systematic study of holomorphic automorphisms of $\mathbb{C}^{n}$ started in the 1980s with the paper of Rosay-Rudin [19]. An important role is played by the so-called holomorphic shears and overshears. We will focus here on the two-dimensional situation.

Communicated by Alexander Isaev.

Dedicated to the memory of Mikael Passare.

R. B. Andrist ( $\square)$

Fachbereich C - Mathematik, Bergische Universität Wuppertal, Wuppertal, Germany

e-mail: rafael.andrist@math.uni-wuppertal.de

F. Kutzschebauch

Institute of Mathematics, University of Bern, Bern, Switzerland

e-mail: frank.kutzschebauch@math.unibe.ch

A. Lind

The Department of Science Education and Mathematics, Mid Sweden University, Sundsvall, Sweden e-mail: andreas.lind@miun.se 
Definition 1.1 A map $S_{g}: \mathbb{C}^{2} \rightarrow \mathbb{C}^{2}$ of the form

$$
S_{g}(x, y)=(x, y+g(x))
$$

(or with the role of 1 st and 2 nd coordinates exchanged) is called a shear map, where $g: \mathbb{C} \rightarrow \mathbb{C}$ is a holomorphic function. The shear group $S\left(\mathbb{C}^{2}\right)$ is the group generated by these shear maps.

Definition 1.2 A map $O_{f, g}: \mathbb{C}^{2} \rightarrow \mathbb{C}^{2}$ of the form

$$
O_{f, g}(x, y)=\left(x, e^{f(x)} \cdot y+g(x)\right)
$$

(or with the role of 1st and 2nd coordinates exchanged) is called an overshear map, where $f, g: \mathbb{C} \rightarrow \mathbb{C}$ are holomorphic functions. The overshear group OS $\left(\mathbb{C}^{2}\right)$ is the group generated by these overshear maps.

The structure of the overshear group was investigated by Ahern and Rudin [1] who obtained a decomposition of $O S\left(\mathbb{C}^{2}\right)$ as an amalgamated product of the affine group and the group $\mathcal{E}:=\left\{(x, y) \mapsto(a x+b, \gamma(x) \cdot y+f(x)): a \in \mathbb{C}^{*}, b \in \mathbb{C}, \gamma \in\right.$ $\left.\mathcal{O}^{*}(\mathbb{C}), f \in \mathcal{O}(\mathbb{C})\right\}$, a result which is fully analogous to the algebraic case.

Andersén [2] proved in 1990 that $S\left(\mathbb{C}^{n}\right)$ is dense (in compact-open topology) in the group of holomorphic automorphisms $\operatorname{Aut}_{\omega}\left(\mathbb{C}^{n}\right)$ which preserve the form $\omega=$ $\mathrm{d} z_{1} \wedge \cdots \wedge \mathrm{d} z_{n}$. Moreover, he showed that $S\left(\mathbb{C}^{n}\right) \subsetneq$ Aut $_{\omega}\left(\mathbb{C}^{n}\right)$ for $n \geq 2$. Two years later Andersén and Lempert [3] showed that the so-called overshear group $O\left(\mathbb{C}^{n}\right)$ is dense in $\operatorname{Aut}\left(\mathbb{C}^{n}\right)$.

The results of Andersén-Lempert and Ahern-Rudin show that the overshear group is small enough to admit some algebraic description, but large enough to be dense in the group of holomorphic automorphisms of $\mathbb{C}^{2}$, whereas Aut $\left(\mathbb{C}^{2}\right)$ itself seems to be too complicated to allow a nice algebraic description.

Danielewski surfaces have been studied intensively in affine algebraic geometry. With $\mathbb{C}^{2}$ they share the property of having an enormously big automorphism group; on the other hand, they have nontrivial topology. Thus they provide other two-dimensional examples of extremely flexible objects. Their algebraic automorphism group is well understood, whereas the holomorphic automorphism group seems to be more complicated, exactly as is it the case for affine two-space $\mathbb{C}^{2}$.

Definition 1.3 Given a non-constant polynomial $p(z) \in \mathbb{C}[z]$ with only simple zeros,

$$
D_{p}:=\left\{(x, y, z) \in \mathbb{C}^{3}: x \cdot y=p(z)\right\} \subset \mathbb{C}^{3}
$$

with the complex structure induced from $\mathbb{C}^{3}$ is called a Danielewski surface. In the following we denote by $n$ the degree of $p$.

In the present paper we continue the study of the holomorphic automorphism group of Danielewski surfaces started in [11] by further investigating the subgroup introduced in that paper (called the overshear group in analogy to the $\mathbb{C}^{2}$ case) which was shown 
to be dense (in compact-open topology) in the identity component of the holomorphic automorphism group.

Here come the relevant definitions introduced in [11]:

Definition 1.4 A map $O_{f, g}: D_{p} \rightarrow D_{p}$ of the form

$$
O_{f, g}(x, y, z)=\left(x, y+\frac{p\left(e^{x f(x)} \cdot z+x g(x)\right)-p(z)}{x}, e^{x f(x)} \cdot z+x g(x)\right)
$$

(or with the role of 1st and 2nd coordinates exchanged, i.e., $I \circ O_{f, g} \circ I$ ) is called an overshear map, where $f, g: \mathbb{C} \rightarrow \mathbb{C}$ are holomorphic functions, and the involution $I$ of $D_{p}$ is the map interchanging $x$ and $y$.

These maps are well-defined maps from $D_{p} \rightarrow D_{p}$ which can actually be continued to a holomorphic map $\mathbb{C}^{3} \rightarrow \mathbb{C}^{3}$. The name overshear comes from the similarity with overshear maps when looking only at the 1st and 3rd coordinate. Because of the relation $O_{f, g} \circ O_{h, k}=O_{f+h, k \cdot e^{x \cdot f}+g}$ the maps are easily seen to be holomorphic automorphisms of $D_{p}$. The group generated by overshears is called the overshear group $\operatorname{OS}\left(D_{p}\right)$.

A family of completely integrable vector fields on Danielewski surfaces such that the Lie algebra generated by them is dense in the Lie algebra of all holomorphic vector fields has been determined by the 2 nd and 3rd author in [11], i.e., Danielewski surfaces enjoy the so-called density property [21,22]. The time-1-maps of the flows of these vector fields are overshears. And as a consequence, the overshear group is a dense subgroup of the identity component of the group of holomorphic automorphisms.

Danielewski surfaces are important examples of Stein manifolds with density property as they (for degree $p$ at least three) are not homogeneous spaces, which provide another large class of Stein manifolds with density property.

Our main result is the following structure theorem:

Theorem 1 (5.1) Let $D_{p}$ be a Danielewski surface and assume that $\operatorname{deg}(p) \geq 4$. Then the overshear group, $O S\left(D_{p}\right)$, is a free product of $O_{1}$ and $O_{2}$, where $O_{1}$ is generated by $\mathrm{O}_{f, g}$ and $\mathrm{O}_{2}$ generated by $\mathrm{I} \circ \mathrm{O}_{f, g} \circ \mathrm{I}$.

We would like to mention the difference from our structure theorem to the one of Ahern-Rudin, where the amalgamated product is not free. Both structure theorems, the one of Ahern-Rudin and ours, generalize the well-known algebraic results of Jung [8] and van der Kulk [20] concerning polynomial automorphisms of $\mathbb{C}^{2}$ and of Makar-Limanov [14,16,17] concerning polynomial automorphisms of Danielewski surfaces. In the polynomial case a simple degree argument is sufficient whereas in the holomorphic case one has to use Nevanlinna theory. Another complication compared to the algebraic setting arises by the fact that there are nowhere vanishing holomorphic functions whereas in the algebraic case these are constants. Ahern and Rudin [1] needed quite subtle estimates to deal with this situation in $\mathbb{C}^{2}$, whereas we have to use Nevanlinna theory on algebraic surfaces which has been developed by Griffiths and King [6]. We recall the main definitions and facts in Sect. 2. Next we specialize the theory to Danielewski surfaces in Sects. 3 and 4. 
However, the crucial estimate corresponding to that used by Ahern and Rudin in the $\mathbb{C}^{2}$ case was still missing in this theory. This estimate-which might be of independent interest - is our main result concerning Nevanlinna theory. It is an estimate bounding the Nevanlinna characteristic function $\widetilde{m}(\cdot, \cdot)$ of a derivative of a holomorphic function by the Nevanlinna characteristic function of the function itself:

Theorem 2 (4.2) Given a holomorphic function $f: D_{p} \rightarrow \mathbb{C}$, and a vector field $\theta$ which is a lift of a partial derivative on $\mathbb{C}^{2}$, then we have the estimate

$$
\widetilde{m}(\theta(f), r) \leq 14 \cdot \widetilde{m}(f, r)+K(n) \cdot \log (r)+L
$$

for big $r$ outside a set of finite linear measure, where $K(n)$ is an affine polynomial and $L$ is a constant.

In the last section we prove the structure theorem. As an easy application we show that the overshear group on Danielewski surfaces is a proper subgroup of the connected component of the holomorphic automorphism group by providing a concrete example of a holomorphic automorphism which is not a composition of overshears; see Corollary 5.7.

In the case of degree $n=2$ we give an explicit counterexample (see Example 5.9) which shows that this decomposition is not a free product. For the degree $n=3$ the question remains open.

In a forthcoming paper we will apply our structure theorem to classify actions of connected real Lie groups on Danielewski surfaces which act by automorphisms contained in the overshear group. This will be a contribution to Holomorphic Linearization similar to [10], for more details about that problem we refer the reader to $[4,5,7]$. We would also like to mention that the overshear group on Danielewski surfaces has recently been used to obtain results on the holomorphic automorphism group of the spectral unit ball $\Omega M(2 \times 2, \mathbb{C})$ of complex two-by-two matrices, a well-studied object in complex analysis. The connection between these subjects is simple: $\Omega M(2 \times 2, \mathbb{C})$ admits a holomorphic fibration (over the symmetrized bidisc) whose generic fibers are Danielewski surfaces such that the overshears extend to fiber preserving holomorphic automorphisms of $\Omega M(2 \times 2, \mathbb{C})[12]$.

\section{Nevanlinna Theory for Algebraic Varieties}

In this section we state the results of Griffiths and King [6] that we need.

We use the following notation:

$$
\begin{aligned}
\mathrm{d} & =\partial+\bar{\partial} \\
\mathrm{d}^{c} & =\frac{i}{4 \pi}(\bar{\partial}-\partial)
\end{aligned}
$$

Definition 2.1 Let $M$ be an algebraic complex manifold. A function $\tau: M \rightarrow$ $[-\infty,+\infty)$ is called an exhaustion function, if

(1) $\tau$ is $\mathcal{C}^{\infty}$-smooth except for finitely many logarithmic singularities 
(2) the half spaces $M[r]:=\left\{z \in M: e^{\tau(z)} \leq r\right\}$ are compact for $r \in[0,+\infty)$

A logarithmic singularity of $\tau$ means that in suitable local coordinates one can write $\tau(z)=\log \|z\|+\tilde{\tau}(z)$, where $\tilde{\tau}$ is $\mathcal{C}^{\infty}$-smooth.

Definition 2.2 Let $M$ be an algebraic complex manifold of dimension $m$. An exhaustion function $\tau: M \rightarrow[-\infty,+\infty)$ is called a special exhaustion function, if

(1) $\tau$ has only finitely many critical points

(2) $\operatorname{dd}^{c} \tau \geq 0$, i.e., $\tau$ is plurisubharmonic

(3) $\left(\mathrm{dd}^{c} \tau\right)^{m-1} \not \equiv 0$ on each of the holomorphic tangent spaces to $\partial M[r]$ for all $r \in[0, \infty)$

(4) $\left(\mathrm{dd}^{c} \tau\right)^{m}=0$

Definition 2.3 Let $M$ be an algebraic complex manifold of dimension $m$ with a special exhaustion function $\tau: M \rightarrow[-\infty,+\infty)$, and $\alpha: M \rightarrow \mathbb{C P}^{1}$ a meromorphic function. We introduce the following notation:

$$
\begin{aligned}
\log ^{+}(x) & :=\max \{\log (x), 0\}, x \geq 0 \\
\psi & :=\operatorname{dd}^{c} \tau, \text { the Levi form of } \tau \\
\eta & :=\mathrm{d}^{c} \tau \wedge \psi^{m-1}, \text { the volume form on } \partial M[r] \\
m(\alpha, r) & :=\int_{\partial M[r]} \log ^{+}\left(\frac{1}{|\alpha|^{2}}\right) \eta, r \geq 0
\end{aligned}
$$

For a divisor $D$ on $M$ we define $D[r]:=D \cap M[r]$ and introduce the following notions, assuming $D$ does not pass through any of the logarithmic singularities of $\tau$ :

$$
\begin{aligned}
n(D, t) & :=\int_{D[t]} \psi^{m-1} \\
N(D, r) & :=\int_{0}^{r} \frac{n(D, t)}{t} \mathrm{~d} t, \text { the counting function }
\end{aligned}
$$

If $D$ passes through a logarithmic singularity of $\tau$, these definitions need to be refined using Lelong numbers, as discussed in $§ 1 \mathrm{~d}$ of [6].

However, we shall not need this refinement using Lelong numbers in our applications to Danielewski surfaces, since the group of holomorphic automorphisms acts transitively on them [11] and we can therefore always assume that $D$ does not pass through a logarithmic singularity. For meromorphic $\alpha: M \rightarrow \mathbb{C P}^{1}$ and $a \in \mathbb{C P}^{1}$ we denote by $D_{a}$ the divisor $\{\alpha(z)=a\}$.

Now one can formulate Jensen's theorem in these terms:

Proposition 2.4 With the previously defined notation, the following equation holds:

$$
N\left(D_{0}, r\right)+m(\alpha, r)=N\left(D_{\infty}, r\right)+m(1 / \alpha, r)+O(1) .
$$


Let

$$
T_{0}(\alpha, r):=N\left(D_{\infty}, r\right)+m(1 / \alpha, r) .
$$

$T_{0}$ is called the Nevanlinna characteristic function of $\alpha$.

It satisfies the following properties:

(1) $T_{0}\left(\alpha_{1} \alpha_{2}, r\right) \leq T_{0}\left(\alpha_{1}, r\right)+T_{0}\left(\alpha_{2}, r\right)$

(2) $T_{0}\left(\alpha_{1}+\alpha_{2}, r\right) \leq T_{0}\left(\alpha_{1}, r\right)+T_{0}\left(\alpha_{2}, r\right)+O(1)$

(3) $T_{0}\left(\alpha_{1}-a, r\right)=T_{0}\left(\alpha_{1}, r\right)+O(1), a \in \mathbb{C}$

(4) $T_{0}\left(1 / \alpha_{1}, r\right)=T_{0}\left(\alpha_{1}, r\right)+O(1)$

where $O(1)$ refers to a bounded term with respect to $r$.

To simplify notation, we define, with the divisor $D_{a}$ as given before:

$$
N(\alpha, r):=N\left(D_{0}, r\right) N(1 / \alpha, r):=N\left(D_{\infty}, r\right)
$$

Example 2.5 For $M=\mathbb{C}^{n}$, a special exhaustion function is given by $\tau_{0}: \mathbb{C}^{n} \rightarrow$ $[-\infty,+\infty), z \mapsto \log (|z|)$. A special exhaustion function for a Danielewski surface will be given later and reduced to this special case-it is therefore worth calculating explicitly the involved quantities for $\mathbb{C}^{2}$ : It is obvious that $\tau_{0}$ is an exhaustion function with a logarithmic singularity in 0 only. In the following we write $z=(x, y)$ $\in \mathbb{C}^{2}$.

(1) Outside the logarithmic singularity there are no critical points of $\tau_{0}$ :

$$
\begin{aligned}
\bar{\partial} \tau_{0} & =\frac{1}{2} \frac{x \mathrm{~d} \bar{x}+y \mathrm{~d} \bar{y}}{x \bar{x}+y \bar{y}} \\
\partial \tau_{0} & =\frac{1}{2} \frac{\bar{x} \mathrm{~d} x+\bar{y} \mathrm{~d} y}{x \bar{x}+y \bar{y}}
\end{aligned}
$$

(2) $\quad \mathrm{dd}^{c} \tau_{0}=\frac{i}{2 \pi} \partial \bar{\partial} \tau_{0}=\frac{i}{2 \pi} \frac{y \bar{y} \mathrm{~d} x \wedge \mathrm{d} \bar{x}+x \bar{x} \mathrm{~d} y \wedge \mathrm{d} \bar{y}-x \bar{y} \mathrm{~d} y \wedge \mathrm{d} \bar{x}-y \bar{x} \mathrm{~d} x \wedge \mathrm{d} \bar{y}}{2(x \bar{x}+y \bar{y})^{2}} \geq 0$

(3) $\partial M[r]=\left\{x \bar{x}+y \bar{y}=r^{2}\right\}$ and the holomorphic tangent space in $(x, y)$ is given by the relation $\bar{x} \mathrm{~d} x+\bar{y} \mathrm{~d} y=0$. Therefore,

$$
\mathrm{dd}^{c} \tau_{0}=\frac{i}{2 \pi r^{2}} \mathrm{~d} y \wedge \mathrm{d} \bar{y} \not \equiv 0 \text { on the holomorphic tangent space. }
$$

(4) $\left(\mathrm{dd}^{c} \tau_{0}\right)^{2}=0$ can be seen by explicit calculation.

For the volume form $\eta_{0}$ we get: 


$$
\begin{aligned}
\eta_{0}:=\mathrm{d}^{c} \tau_{0} \wedge \mathrm{dd}^{c} \tau_{0}= & \frac{1}{8 \pi^{2}} \partial \bar{\partial} \tau_{0} \wedge(\partial-\bar{\partial}) \tau_{0} \\
= & \frac{1}{(4 \pi)^{2}(x \bar{x}+y \bar{y})^{2}}(\bar{y} \mathrm{~d} x \wedge \mathrm{d} \bar{x} \wedge \mathrm{d} y-y \mathrm{~d} x \wedge \mathrm{d} \bar{x} \wedge \mathrm{d} \bar{y} \\
& +\bar{x} \mathrm{~d} x \wedge \mathrm{d} y \wedge \mathrm{d} \bar{y}-x \mathrm{~d} \bar{x} \wedge \mathrm{d} y \wedge \mathrm{d} \bar{y})
\end{aligned}
$$

It is rotation invariant since $\tau_{0}$ is, and scales such that $\eta_{0}(r x, r y)=\eta_{0}(x, y)$, and in particular we have that

$$
\int_{r S^{3}} \eta_{0}=r^{3} \cdot \int_{S^{3}} \eta_{0}=2 r^{3}
$$

\section{Nevanlinna Theory for Danielewski Surfaces}

For a Danielewski surface $D_{p}$ we define a projection $\pi: D_{p} \rightarrow \mathbb{C}^{2}$ as

$$
\pi(x, y, z)=(x+y, z)
$$

and for the special exhaustion function we choose

$$
\begin{aligned}
& \tau: D_{p} \rightarrow[-\infty,+\infty) \\
& (x, y, z) \mapsto \log (|\pi(x, y, z)|)
\end{aligned}
$$

Lemma 3.1 This function $\tau$ defined above is a special exhaustion function in the sense of Definition 2.2.

Proof $\log (|\pi(x, y, z)|)$ can only have logarithmic singularities when $\pi(x, y, z)=$ $(0,0)$, i.e., $x+y=0, z=0$ and therefore $x \cdot y=p(0)$ which happens only for two points. Let $\tau_{0}: \mathbb{C}^{2} \rightarrow[-\infty,+\infty)$ be the special exhaustion function defined in Example 2.5. We can then write $\tau=\tau_{0} \circ \pi$. The projection $\pi$ is linear, therefore the results of calculations are the same as in the case of $\mathbb{C}^{2}$, when in the end $(x, y)$ is formally replaced by $(x+y, z)$. Now we check the properties required by Definition 2.2:

(1) Critical points can occur only in case of $x+y=0$ and $z=0$ :

$$
\begin{aligned}
\bar{\partial} \tau & =\frac{1}{2} \frac{(x+y)(\mathrm{d} \bar{x}+\mathrm{d} \bar{y})+z \mathrm{~d} \bar{z}}{(x+y)(\bar{x}+\bar{y})+z \bar{z}} \\
\partial \tau & =\frac{1}{2} \frac{(\bar{x}+\bar{y})(\mathrm{d} x+\mathrm{d} y)+\bar{z} \mathrm{~d} z}{(x+y)(\bar{x}+\bar{y})+z \bar{z}}
\end{aligned}
$$

So there are in fact no critical points at all (outside the two logarithmic singularities).

(2) $\mathrm{dd}^{c} \tau \geq 0$ follows directly from the linearity of $\pi$. 
(3) $\partial M[r]=\left\{(x+y)(\bar{x}+\bar{y})+z \bar{z}=r^{2}, x \cdot y=p(z)\right\}$ and the holomorphic tangent space in $(x, y, z)$ is given by the relations $(\bar{x}+\bar{y})(\mathrm{d} x+\mathrm{d} y)+\bar{z} \mathrm{~d} z=0$ and $y \mathrm{~d} x+x \mathrm{~d} y=p^{\prime}(z) \mathrm{d} z$, hence:

$$
\operatorname{dd}^{c} \tau=\frac{i}{2 \pi r^{2}} \mathrm{~d} z \wedge \mathrm{d} \bar{z} \not \equiv 0 \text { on the holomorphic tangent space. }
$$

(4) $\left(\mathrm{dd}^{c} \tau\right)^{2}=0$ follows directly from the linearity of $\pi$.

Now we want to write out more explicitly the Nevanlinna characteristic function for a holomorphic function $f: D_{p} \rightarrow \mathbb{C}$ where the expression reduces to

$$
\begin{aligned}
T_{0}(f, r) & =m(1 / f, r)=\int_{\partial D_{p}[r]} \log ^{+}\left(|f|^{2}\right) \eta \\
& =\int_{\partial D_{p}[r]} \log ^{+}\left(|f|^{2}\right) \pi^{*}\left(\eta_{0}\right) \\
& =\int_{\pi^{-1}\left(r S^{3}\right)} \log ^{+}|f|^{2} \pi^{*}\left(\eta_{0}\right)
\end{aligned}
$$

In order to apply this Nevanlinna characteristic function to overshears in the next section, we need to estimate derivatives with respect to $r$. This can be calculated easier in an even more explicit form. First we need to investigate the projection $\pi$ a bit more.

Ramification points of $\pi$ : Given $(a, b) \in \mathbb{C}^{2}$, we look for the pre-images $(x, y, z) \in$ $D_{p}$, i.e., $(x, y, z) \in \mathbb{C}^{3}$ such that

$$
\begin{aligned}
a & =x+y \\
b & =z \\
p(z) & =x \cdot y
\end{aligned}
$$

This obviously leads to a quadratic equation, symmetric in $x$ and $y$. Therefore, ramification points occur exactly if $x=y$, otherwise the covering has 2 sheets. For $(a, b) \in \mathbb{C}^{2}$ this means that $p(b)=\frac{a^{2}}{4}$. We cut out these points in the following way: For every $(a, b) \in \mathbb{C}^{2}$ satisfying $p(b)=\frac{a^{2}}{4}$, we cut along the real $a$-line towards $-\infty$. The set of points we cut out is denoted by $C$, a set of real dimension 3 . Now, $\pi: \pi^{-1}\left(\mathbb{C}^{2} \backslash C\right) \rightarrow \mathbb{C}^{2} \backslash C$ is an unbranched 2 -sheeted covering, and $\mathbb{C}^{2} \backslash C$ is simply connected. Since a line is always either tangential or transversal to a sphere, the intersection of $C$ with $r S^{3} \subset \mathbb{C}^{2}$ is always a set of real dimension 2 and therefore of measure zero with respect to $\eta_{0}$, hence its removal does not affect the integral. By $s_{j}: \mathbb{C}^{2} \backslash C \rightarrow D_{p}(j=1,2)$ we now denote the sections corresponding to this restricted unbranched covering s.t. $\pi \circ s_{j}=\mathrm{id}$. 


$$
\begin{aligned}
m(1 / f, r) & =\int_{\pi^{-1}\left(r S^{3}\right)} \log ^{+}|f|^{2} \pi^{*}\left(\eta_{0}\right) \\
& =\int_{r S^{3} \backslash C} \sum_{j=1}^{2} \log ^{+}\left|f \circ s_{j}\right|^{2} \eta_{0}
\end{aligned}
$$

Definition 3.2 Ahern and Rudin [1] used a slightly different definition of the Nevanlinna characteristic function for holomorphic $f: \mathbb{C}^{n} \rightarrow \mathbb{C}$, namely

$$
\widetilde{m}(f, r):=\int_{S^{2 n-1} \subseteq \mathbb{C}^{n}} \log ^{+}|f(r \zeta)| \mathrm{d} \sigma(\zeta),
$$

where $\sigma$ is a finite rotational invariant Borel measure on the sphere $S^{2 n-1}$.

By choosing an appropriate normalization for the measure $\sigma$ we can arrange that on $\mathbb{C}^{n}$ the following relation holds:

$$
r^{2 n-1} \cdot \widetilde{m}(f, r)=m(1 / f, r)
$$

We choose this as a definition for $\widetilde{m}(f, r)$ on a Danielewski surface $(n=2)$. All properties so far derived for $m(f, r)$ are inherited by $\widetilde{m}(f, r)$, except the growth rate in $r$ which gets modified by $r^{3}$.

Lemma 3.3 Let $f, g: D_{p} \rightarrow \mathbb{C}$ be holomorphic functions. Then their Nevanlinna characteristics satisfy the following properties:

(1) $\widetilde{m}(f, r)-\widetilde{m}(g, r)+O(1) \leq \widetilde{m}(f+g, r) \leq \widetilde{m}(f, r)+\widetilde{m}(g, r)+O(1)$

(2) $\tilde{m}(f \cdot g, r) \leq \widetilde{m}(f, r)+\widetilde{m}(g, r)+O(1)$

(3) $\widetilde{m}(1 / f, r) \leq \widetilde{m}(f, r)+O(1)$

Proof The first two properties follow directly from

$$
\log (x)=\log ^{+}(x)-\log ^{+}(1 / x), x \in \mathbb{R}_{+}^{*}
$$

and then thereby from inherited inequalities of the logarithm:

$$
\begin{aligned}
& \log ^{+}|z+w| \leq \log ^{+}(2 \max \{|z|,|w|\}) \leq \log ^{+}|z|+\log |w|+\log 2, z, w, z+w \in \mathbb{C}^{*} \\
& \log ^{+}|z w| \leq \log ^{+}|z|+\log ^{+}|w|, z, w \in \mathbb{C}^{*}
\end{aligned}
$$

For property 3 we need Jensen's formula (7):

$$
\begin{aligned}
r^{3} \widetilde{m}(1 / f, r) & =m(f, r)=T_{0}(1 / f, r) \\
& =T_{0}(f, r)+O(1)=m(1 / f, r)+N(1 / f, r)+O(1)
\end{aligned}
$$

Now observe that $\frac{1}{r^{3}} N(1 / f, r)$ is of order $O(1)$, since the counting function of such a divisor associated to $1 / f$ has support at most in a hypersurface. 
Using these elementary properties we are now able to prove

Proposition 3.4 Let $f: D_{p} \rightarrow \mathbb{C}$ be a meromorphic function, and let

$$
R_{d}(z, f(z))=a_{0}(z)+a_{1}(z) f(z)+\cdots+a_{d}(z)(f(z))^{d},
$$

where $a_{i}: D_{p} \rightarrow \mathbb{C}$ are meromorphic, and assume that $a_{d} \not \equiv 0$. Then

$$
\widetilde{m}\left(R_{d}(z, f(z)), r\right)=d \cdot \widetilde{m}(f, r)+\sum_{i=0}^{d} O\left(\widetilde{m}\left(a_{i}, r\right)\right)+O(1)
$$

In the case of meromorphic functions defined on $\mathbb{C}$, this proposition was first proved by Mohon'ko [18]. Mohon'ko's paper is in Russian, but an English proof can be found in [13]. We adapt the same proof to our more general situation.

\section{Lemma 3.5 Let}

$$
A(z, f):=\left(\varphi_{1}(z) f+\cdots+\varphi_{d-1}(z) f^{d-1}+f^{d}\right) f^{d-2}
$$

be a polynomial in $f$ with meromorphic coefficients. Then there exist $u_{0}, \ldots, u_{d-1}$, $q_{0}, \ldots, q_{d-2}$ which are polynomials in $\varphi_{1}, \ldots, \varphi_{d-1}$ with constant coefficients, such that

$$
B(z, f):=u_{0}(z)+\cdots+u_{d-1}(z) f^{d-1}
$$

satisfies

$$
(B(z, f))^{2}=A(z, f)+\sum_{i=0}^{d-2} q_{i}(z) f^{i}
$$

The proof of Lemma 3.5 can be found in either [18] or [13], but it is not so hard to see that $u_{d-1} \equiv 1, u_{d-2}(z)=\frac{1}{2} \varphi_{d-1}(z)$,

$$
u_{d-k}(z)=\frac{1}{2} \cdot\left(\varphi_{d-k+1}(z)-u_{d-k+1}^{2}(z)\right), \quad k=3, \ldots, d
$$

and

$$
q_{i}(z)=u_{0}(z) u_{i}(z)+u_{1}(z) u_{i-1}(z)+\cdots+u_{i}(z) u_{0}(z)
$$

solve the problem. 
Proof of Proposition 3.4.

$$
\begin{aligned}
\widetilde{m}\left(\sum_{i=0}^{d} a_{i} f^{i}, r\right) & \leq \widetilde{m}\left(f \cdot \sum_{i=1}^{d} a_{i} f^{i-1}, r\right)+\widetilde{m}\left(a_{0}, r\right)+O(1) \\
& \leq \widetilde{m}(f, r)+\widetilde{m}\left(\sum_{i=1}^{d} a_{i} f^{i-1}, r\right)+\widetilde{m}\left(a_{0}, r\right)+O(1)
\end{aligned}
$$

By induction

$$
\widetilde{m}\left(\sum_{j=0}^{d} a_{j} f^{j}, r\right) \leq d \cdot \widetilde{m}(f, r)+\sum_{i=0}^{d} O\left(\widetilde{m}\left(a_{i}, r\right)\right)+O(1) .
$$

Conversely, assume first that $d=1$. Then

$$
\widetilde{m}(f, r)=\widetilde{m}\left(\frac{R_{1}-a_{0}}{a_{1}}, r\right) \leq \widetilde{m}\left(R_{1}, r\right)+\widetilde{m}\left(a_{0}, r\right)+\widetilde{m}\left(a_{1}, r\right)+O(1) .
$$

Rearranging this inequality proves the proposition for $d=1$.

Now, assume that the proposition has been proved for all polynomials $P(z, f)$, as in the statement, of degree $s \leq d-1$ in $f$. That is,

$$
\widetilde{m}(P(z, f), r)=s \cdot \widetilde{m}(f, r)+\sum_{j=0}^{d-1} O\left(\widetilde{m}\left(a_{j}, r\right)\right)+O(1) .
$$

Observe that

$$
\frac{R_{d}-a_{0}}{a_{d}} f^{d-2}=\left(\varphi_{1} f+\cdots+\varphi_{d-1} f^{d-1}+\varphi_{d} f^{d}\right) \cdot f^{d-2},
$$

where $\varphi_{j}=a_{j} / a_{d}$ for $j=0, \ldots, d-1$. Using Lemma 3.5 we see that

$$
(B(z, f))^{2}=\frac{R_{d}-a_{0}}{a_{d}} f^{d-2}+\sum_{i=0}^{d-2} q_{i}(z) f^{i}
$$

where the degree of $B(z, f)$ in $f$ is $d-1$. By the induction hypothesis we get

$$
\begin{aligned}
\widetilde{m}\left((B(z, f))^{2}, r\right) & =2 \cdot \widetilde{m}(B(z, f), r) \\
& =2(d-1) \cdot \widetilde{m}(f, r)+\sum_{i=0}^{d-1} O\left(\widetilde{m}\left(a_{i}, r\right)\right)+O(1) .
\end{aligned}
$$

On the other hand, 


$$
\widetilde{m}\left((B(z, f))^{2}, r\right) \leq(d-2) \widetilde{m}(f, r)+\widetilde{m}\left(\frac{R_{d}-a_{0}}{a_{d}}, r\right)+\sum_{i=0}^{d-2} \widetilde{m}\left(q_{i}, r\right)+O(1) .
$$

Looking at the proof of Lemma 3.5 and performing an obvious subtraction gives

$$
\widetilde{m}\left(R_{d}, r\right) \geq d \cdot \tilde{m}(f, r)+\sum_{i=0}^{d} O\left(\widetilde{m}\left(a_{i}, r\right)\right)+O(1) .
$$

Now we have shown both inequalities.

Corollary 3.6 Let $q \in \mathbb{C}[z]$ be a polynomial, and $f: D_{p} \rightarrow \mathbb{C}$ a holomorphic function. Then

$$
\widetilde{m}(q \circ f, r)=\operatorname{deg}(q) \cdot \tilde{m}(f, r)+O(1)
$$

Proposition 3.7 Let $f: D_{p} \rightarrow \mathbb{C}$ be a holomorphic function and $g: \mathbb{C} \rightarrow \mathbb{C}$ transcendental. Then

$$
\lim _{r \rightarrow \infty} \frac{T_{0}(g \circ f, r)}{T_{0}(f, r)}=\lim _{r \rightarrow \infty} \frac{\widetilde{m}(g \circ f, r)}{\widetilde{m}(f, r)}=\infty
$$

Proof (1) Let $g: \mathbb{C} \rightarrow \mathbb{C}$ be transcendental. By Picard's theorem we can assume without loss of generality that $g$ has infinitely many zeros; otherwise, we consider instead $z \mapsto g(z)+c, c \in \mathbb{C}^{*}$, and note that $T_{0}(\alpha, r)=T_{0}(\alpha+c, r)+O(1)$. Let $\left\{w_{j}\right\}_{j=1}^{\infty}$ be the zeros of $g$.

(2) Claim:

$$
\widetilde{m}\left(\prod_{j=1}^{n} \frac{1}{f-w_{j}}, r\right) \leq \tilde{m}\left(\frac{1}{g \circ f}, r\right)+O(1)
$$

Proof:

$$
\begin{aligned}
& \widetilde{m}\left(\frac{1}{g \circ f}, r\right)-\widetilde{m}\left(\prod_{j=1}^{n} \frac{1}{f-w_{j}}, r\right) \\
& =2 \int_{\pi^{-1}\left(r S^{3}\right)}\left(\log ^{+}\left|\frac{1}{(g \circ f)(z)}\right|-\log ^{+}\left|\prod_{j=1}^{n} \frac{1}{f(z)-w_{j}}\right|\right) \eta(z)
\end{aligned}
$$

Recall that $\eta$ is a positive volume form. Hence a negative integrand would imply $\left|\prod_{j=1}^{n} \frac{1}{f(z)-w_{j}}\right|>1$. The set $\Omega \subseteq \mathbb{C}$ defined by $\left\{w \in \mathbb{C}:\left|\prod_{j=1}^{n}\left(w-w_{j}\right)\right|<1\right\}$ is bounded, hence there exists an $A>0:|g \circ f| \leq A$ on $\bar{\Omega}$. The integrand is certainly smaller if we integrate only over the set such that the integrand is negative, i.e., we continue with the estimates: 


$$
\begin{aligned}
& \geq 2 \int_{f^{-1}(\Omega) \cap \pi^{-1}\left(r S^{3}\right)}\left(\log ^{+}\left|\frac{1}{(g \circ f)(z)}\right|-\log ^{+}\left|\prod_{j=1}^{n} \frac{1}{f(z)-w_{j}}\right|\right) \eta(z) \\
& \geq 2 \underset{\left\{z \in D_{p}: f(z) \in \Omega\right\} \cap \pi^{-1}\left(r S^{3}\right)}{2} \log \left|\frac{1}{g \circ f}\right| \eta \geq 2 \log \frac{1}{A},
\end{aligned}
$$

where $A$ depends on $n$, but not on $r$, and this proves the claim.

(3) We state two facts about $N(\cdot, r)$; they are immediate consequences of the fact that this is the counting function of a divisor.

$$
\begin{array}{r}
N\left(\frac{1}{g \circ f}, r\right) \geq N\left(\prod_{j=1}^{n} \frac{1}{f-w_{j}}, r\right) \\
N\left(\prod_{j=1}^{n} \frac{1}{f-w_{j}}, r\right)=\sum_{j=1}^{n} N\left(\frac{1}{f-w_{j}}, r\right)
\end{array}
$$

(4) By Jensen's formula (7) we have:

$$
\begin{aligned}
& N\left(\prod_{j=1}^{n} \frac{1}{f-w_{j}}, r\right)+\widetilde{m}\left(\prod_{j=1}^{n} \frac{1}{f-w_{j}}, r\right) \\
& =N(\underbrace{\left.\prod_{j=1}^{n}\left(f-w_{j}\right), r\right)}_{=0}+\widetilde{m}\left(\prod_{j=1}^{n}\left(f-w_{j}\right), r\right)+O(1)
\end{aligned}
$$

as well as

$$
\begin{aligned}
& N\left(\frac{1}{f-w_{j}}, r\right)+\widetilde{m}\left(\frac{1}{f-w_{j}}, r\right) \\
& =\underbrace{N\left(f-w_{j}, r\right)}_{=0}+\widetilde{m}\left(f-w_{j}, r\right)+O(1)
\end{aligned}
$$

Summing up the last equation over $j$ and using Jensen's formula again, we obtain:

$$
\begin{aligned}
& \sum_{j=1}^{n} N\left(\frac{1}{f-w_{j}}, r\right)+\sum_{j=1}^{n} \tilde{m}\left(\frac{1}{f-w_{j}}, r\right) \\
& \quad=\sum_{j=1}^{n} \tilde{m}\left(f-w_{j}, r\right)+O(1)=\widetilde{m}\left(\prod_{j=1}^{n}\left(f-w_{j}\right), r\right)+O(1) \\
& \quad=N\left(\prod_{j=1}^{n} \frac{1}{f-w_{j}}, r\right)+\widetilde{m}\left(\prod_{j=1}^{n} \frac{1}{f-w_{j}}, r\right)+O(1)
\end{aligned}
$$




$$
\text { which implies } \sum_{j=1}^{n} \widetilde{m}\left(\frac{1}{f-w_{j}}, r\right)=\widetilde{m}\left(\prod_{j=1}^{n} \frac{1}{f-w_{j}}, r\right)+O(1)
$$

The inequality (9) and equation (14) together finally give:

$$
\widetilde{m}\left(\frac{1}{g \circ f}, r\right) \geq \sum_{j=1}^{n} \widetilde{m}\left(\frac{1}{f-w_{j}}, r\right)+O(1)
$$

(5) Summing up inequalities (15) and (10), we obtain:

$$
T_{0}\left(\frac{1}{g \circ f}, r\right) \geq \sum_{j=1}^{n} T_{0}\left(\frac{1}{f-w_{j}}, r\right)+O(1)
$$

and by Jensen's formula, this is equivalent to

$$
\begin{aligned}
T_{0}(g \circ f, r) & \geq \sum_{j=1}^{n} T_{0}\left(f-w_{j}, r\right)+O(1) \\
& =n \cdot T_{0}(f, r)+O(1)
\end{aligned}
$$

Therefore, we have for all $n \in \mathbb{N}$ :

$$
\lim _{r \rightarrow \infty} \frac{T_{0}(g \circ f, r)}{T_{0}(f, r)} \geq n
$$

Lemma 3.8 Let $p$ be a polynomial of degree $n, D_{p}$ the corresponding Danielewski surface. By $x, y, z: D_{p} \rightarrow \mathbb{C}$ we refer to the coordinate functions of $\mathbb{C}^{3}$, restricted to $D_{p}$. Then the growth of the Nevanlinna characteristic function can be described as follows:

(1) $\lim _{r \rightarrow \infty} \frac{\widetilde{m}(x, r)}{\widetilde{m}(y, r)}=1$

(2) $\lim _{r \rightarrow \infty} \frac{\widetilde{m}(x, r)}{\widetilde{m}(z, r)}=\lim _{r \rightarrow \infty} \frac{\widetilde{m}(y, r)}{\widetilde{m}(z, r)}=\frac{n}{2}$

(3) $O(\widetilde{m}(z, r))=O(\log r)$

Proof The relations between the three Nevanlinna characteristic functions follow directly from $x \cdot y=p(z)$ and Proposition 3.4. It is therefore sufficient to calculate the asymptotic behavior of $\widetilde{m}(z, r)$ which is, using the covering $\pi: D_{p} \rightarrow \mathbb{C}^{2}$, twice the Nevanlinna characteristic function of a coordinate in $\mathbb{C}^{2}$ :

$$
\tilde{m}(f, r)=\int_{S^{3} \subseteq \mathbb{C}^{2}} \log ^{+}|f(r \zeta)| \mathrm{d} \sigma(\zeta) \leq \sigma\left(S^{3}\right) \cdot \log (r), \quad r \geq 1
$$

with $f\left(z_{1}, z_{2}\right)=z_{1}$. 


\section{Nevanlinna Characteristic Function of Derivatives}

Ahern and Rudin [1] showed

Proposition 4.1 Let $f: \mathbb{C}^{k} \rightarrow \mathbb{C}$ be holomorphic. Then

$$
\tilde{m}_{\mathbb{C}^{k}}\left(\frac{\partial f}{\partial z_{j}}, r\right) \leq 3 \cdot \tilde{m}_{\mathbb{C}^{k}} f r+k \log r
$$

The objective for the rest of this section is to derive a similar estimate in the case of Danielewski surfaces. Let $(a, b) \in \mathbb{C}^{2}$ be coordinates on $\mathbb{C}^{2}$. Using the branched covering $\pi: D_{p} \rightarrow \mathbb{C}^{2}$ to lift the vector fields $\frac{\partial}{\partial a}$ and $\frac{\partial}{\partial b}$ we get the following meromorphic vector fields on $D_{p}$ :

$$
\theta_{1}=\frac{x}{x-y} \frac{\partial}{\partial x}-\frac{y}{x-y} \frac{\partial}{\partial y}
$$

and

$$
\theta_{2}=\frac{p^{\prime}(z)}{y-x}\left(\frac{\partial}{\partial x}-\frac{\partial}{\partial y}\right)+\frac{\partial}{\partial z} .
$$

Since $\theta_{i}$ correspond to partial derivatives, we want to estimate, in the spirit of Proposition 4.1, $\widetilde{m}\left(\theta_{i}(f), r\right)$ to generalize Ahern and Rudin's proposition to Danielewski surfaces.

Theorem 4.2 Given a holomorphic function $f: D_{p} \rightarrow \mathbb{C}$, and a vector field $\theta$ which is a lift of a partial derivative on $\mathbb{C}^{2}$, then we have the estimate

$$
\widetilde{m}(\theta(f), r) \leq 14 \cdot \widetilde{m}(f, r)+K(n) \cdot \log (r)+L
$$

for big $r$ outside a set of finite linear measure, where $K(n)$ is an affine polynomial and $L$ is a constant.

The proof of Theorem 4.2 contains several steps. First an observation.

Remark 4.3 Recall the involution map $I: D_{p} \rightarrow D_{p}$ defined by $I(x, y, z)=$ $(y, x, z)$. Given a function $f: D_{p} \rightarrow \mathbb{C}$ we can decompose this function in an $I$ invariant and an $I$-anti-invariant part by

$$
f=\frac{f+f \circ I}{2}+\frac{f-f \circ I}{2}=f_{\mathrm{inv}}+f_{\mathrm{anti}},
$$

where $f_{\text {inv }}:=\frac{f+f \circ I}{2}$ is $I$-invariant while $f_{\text {anti }}:=\frac{f-f \circ I}{2}$ is anti-invariant under $I$. Clearly, the invariance respectively anti-invariance means that

$$
f_{\text {inv }} \circ I=f_{\text {inv }}
$$

respectively 


$$
f_{\text {anti }} \circ I=-f_{\text {anti }}
$$

For simplicity, in the following we will write invariant and anti-invariant instead of $I$-invariant and I-anti-invariant.

Lemma 4.4 The Nevanlinna characteristic of $f$ and $f \circ I$ is equal, i.e.,

$$
\widetilde{m}(f, r)=\widetilde{m}(f \circ I, r)
$$

for all $f \in \mathcal{O}\left(D_{p}\right)$.

Proof This is a simple consequence of the definition of $\widetilde{m}(f, r)$ resp. $m(1 / f, r)$ as an integral over $D_{p}$.

The following lemma is trivial:

Lemma 4.5 Every vector field $\theta$ on $D_{p}$ which is a lift from a vector field on $\mathbb{C}^{2}$ is invariant under the involution $I$, i.e., $I_{*} \theta=\theta$.

Given a function $f: D_{p} \rightarrow \mathbb{C}$, decompose it as in Observation 4.3, so $f=f_{\text {inv }}+$ $f_{\text {anti }}$. As every lifted vector field $\theta$ on $D_{p}$ is invariant by Lemma 4.5, we get that $\theta\left(f_{\text {inv }}\right)$ is invariant and that $\theta\left(f_{\text {anti }}\right)$ is anti-invariant. Therefore, by linearity and property 1 in Lemma 3.3 we get

$$
\begin{aligned}
\widetilde{m}(\theta(f), r) & =\widetilde{m}\left(\theta\left(f_{\text {inv }}\right)+\theta\left(f_{\text {anti }}\right), r\right) \\
& \leq \widetilde{m}\left(\theta\left(f_{\text {inv }}\right), r\right)+\widetilde{m}\left(\theta\left(f_{\text {anti }}\right), r\right)+C .
\end{aligned}
$$

By expression (18) we need to estimate the invariant and anti-invariant part separately to prove Theorem 4.2. We prove these different cases in two lemmas.

Lemma 4.6 Let $\theta$ be a lift of a partial derivative on $\mathbb{C}^{2}$ and let $f: D_{p} \rightarrow \mathbb{C}$ be an invariant holomorphic function. Then

$$
\widetilde{m}(\theta(f), r) \leq 3 \widetilde{m}(f, r)+4 \log (r)
$$

for all r outside a set of finite linear measure.

Proof The function $f: D_{p} \rightarrow \mathbb{C}$ defines a holomorphic function $\tilde{f}: \mathbb{C}^{2} \rightarrow \mathbb{C}$ by

$$
\tilde{f}(a, b):=f\left(\pi^{-1}(a, b)\right) .
$$

Then

$$
\widetilde{m}(f, r)=2 \widetilde{m}_{\mathbb{C}^{2}}(\tilde{f}, r),
$$


where $\widetilde{m}_{\mathbb{C}^{2}}(\cdot, \cdot)$ refers to the Nevanlinna characteristic function on the Danielewski surface $\mathbb{C}^{2}$. Indeed, we have

$$
\begin{aligned}
\tilde{m}_{\mathbb{C}^{2}}(\tilde{f}, r) & =\int_{S^{3}} \log ^{+}\left|f\left(\pi^{-1}(r \zeta)\right)\right| d \sigma \\
& =\int_{\bar{x} \in \pi^{-1}\left(r S^{3}\right)} \log ^{+}|f(\bar{x})| d\left(\pi^{*} \sigma\right)(\bar{x})=\frac{1}{2} \widetilde{m}(f, r) .
\end{aligned}
$$

By Proposition 4.1 we know that

$$
\widetilde{m}_{\mathbb{C}^{2}}\left(\frac{\partial \tilde{f}}{\partial z_{i}}, r\right) \leq 3 \widetilde{m}_{\mathbb{C}^{2}}(\tilde{f}, r)+2 \log (r)
$$

for $r$ outside a set of finite linear measure. Assume that $\theta$ is a lift of a partial derivative. Then $\theta(f)$ is invariant by the discussion after Lemma 4.5 and $\theta(f)=\pi^{*}\left(\frac{\partial \tilde{f}}{\partial z_{i}}\right)$. By expression (19) we get that

$$
\widetilde{m}(\theta(f), r)=2 \cdot \widetilde{m}_{\mathbb{C}^{2}}\left(\frac{\partial \tilde{f}}{\partial z_{i}}, r\right) \leq 6 \cdot \widetilde{m}_{\mathbb{C}^{2}}(\tilde{f}, r)+4 \log (r)
$$

by Proposition 4.1, for big $r$ outside a set of finite linear measure. Going back to the characteristic function on $D_{p}$ we get that the right-hand side of inequality (20) equals

$$
3 \cdot \tilde{m}(f, r)+4 \log (r) .
$$

Hence the lemma is proved.

Lemma 4.7 Let $\theta$ be a lift of a partial derivative on $\mathbb{C}^{2}$ and let $f: D_{p} \rightarrow \mathbb{C}$ be an anti-invariant holomorphic function. Then

$$
\widetilde{m}(\theta(f), r) \leq 4 \widetilde{m}(f, r)+E(n) \cdot \log (r)+F
$$

for big $r$ outside a set of finite linear measure, where $E(n)$ is an affine polynomial and $F$ is a constant.

Proof Let $f: D_{p} \rightarrow \mathbb{C}$ be an anti-invariant holomorphic function. Then $(x-y) f$ is invariant. Let $\theta$ be a lift of a partial derivative, and therefore $\theta((x-y) f)$ is invariant by the discussion after Lemma 4.5. By Lemma 4.6 we get that

$$
\widetilde{m}(\theta((x-y) f), r) \leq 3 \cdot \widetilde{m}((x-y) f, r)+4 \log (r)
$$

for all $r$ outside a set of finite linear measure. Since $\theta$ is a vector field it fulfills Leibniz's rule:

$$
\theta((x-y) f)=f \cdot \theta(x-y)+(x-y) \cdot \theta(f)
$$


We get, using property (2) in Lemma 3.3, that

$$
\widetilde{m}(\theta(f), r)=\widetilde{m}\left(\frac{(x-y) \theta(f)}{x-y}, r\right) \leq \widetilde{m}\left(\frac{1}{x-y}, r\right)+\widetilde{m}((x-y) \theta(f), r)
$$

Now use (3) in Lemma 3.3. Then we get that the right-hand side of (23) is less than or equal to

$$
\begin{aligned}
& \widetilde{m}(x-y, r)+\widetilde{m}((x-y) \theta(f), r)+C \\
& \quad=\widetilde{m}(x-y, r)+\widetilde{m}(\theta((x-y) f)-f \cdot \theta(x-y), r)+C,
\end{aligned}
$$

where in the last equality we used expression (22). Using properties (1) and (2) in Lemma 3.3 we get that the right-hand side of (24) is less than or equal to

$$
\widetilde{m}(x-y, r)+\widetilde{m}(\theta((x-y) f), r)+\widetilde{m}(f, r)+\widetilde{m}(\theta(x-y), r)+\tilde{C} .
$$

By equation (21) we get that the right-hand side of (25) is less than or equal to

$$
\begin{aligned}
& \widetilde{m}(x-y, r)+3 \cdot \widetilde{m}((x-y) f, r)+4 \cdot \log (r)+\widetilde{m}(f, r) \\
& \quad+\widetilde{m}(\theta(x-y), r)+\tilde{C}
\end{aligned}
$$

for all $r$ outside a set of finite linear measure. Using 2 from Lemma 3.3 we get that the right-hand side of (26) is less than or equal to

$$
4 \cdot \widetilde{m}(x-y, r)+4 \cdot \log (r)+4 \cdot \widetilde{m}(f, r)+\widetilde{m}(\theta(x-y), r)+\tilde{C} .
$$

By (2) in Lemma 3.3 and by Lemma 3.8 we get that the right-hand side of (27) is less than or equal to

$$
\begin{aligned}
4 \cdot & (2 n \cdot \log (r)+D)+4 \cdot \log (r)+4 \cdot \tilde{m}(f, r)+\tilde{m}(\theta(x-y), r)+\tilde{C} \\
& =(8 n+4) \cdot \log (r)+4 \cdot \tilde{m}(f, r)+\widetilde{m}(\theta(x-y), r)+\tilde{D} .
\end{aligned}
$$

We need to estimate $\widetilde{m}(\theta(x-y), r)$, so we consider two cases, namely $\theta=\theta_{1}$ or $\theta=\theta_{2}$ from equation (16) and equation (17). When $\theta=\theta_{1}$ we get that

$$
\widetilde{m}\left(\left(\frac{x}{x-y} \frac{\partial}{\partial x}-\frac{y}{x-y} \frac{\partial}{\partial y}\right)(x-y), r\right)=\widetilde{m}\left(\frac{x+y}{x-y}, r\right)
$$

By property (1) and (3) in Lemma 3.3 we get that the right-hand side of (29) is less than or equal to

$$
2 \cdot \widetilde{m}(x, r)+2 \cdot \widetilde{m}(y, r)+E=4 n \cdot \log (r)+E,
$$

where we used in the last inequality Lemma 3.8 for big $r$ outside a set of finite linear measure. Combining equations (30) and (28) we get, by following the chain of inequalities from expression (23), that 


$$
\widetilde{m}(\theta(f), r) \leq 4 \cdot \widetilde{m}(f, r)+(12 n+4) \cdot \log (r)+\tilde{E}
$$

for big $r$ outside a set of finite linear measure.

Continuing with the next case, we assume that $\theta=\theta_{2}$. Then we get that $\widetilde{m}(\theta(x-y), r)$ equals

$$
\widetilde{m}\left(\left(\frac{p^{\prime}(z)}{y-x}\left(\frac{\partial}{\partial x}-\frac{\partial}{\partial y}\right)+\frac{\partial}{\partial z}\right)(x-y), r\right)=\widetilde{m}\left(\frac{2 p^{\prime}(z)}{y-x}, r\right)
$$

Using property (1) and (3) in Lemma 3.3, yields that the right-hand side of (32) is less than or equal to

$$
\widetilde{m}\left(\left(2 p^{\prime}(z), r\right)+\widetilde{m}(x, r)+\widetilde{m}(y, r)+E=(3 n-1) \cdot \log (r)+E,\right.
$$

where in the last equality we used Lemma 3.8 and Corollary 3.6 for big $r$ outside a set of finite linear measure. Expression (33) together with expression (28) yields, following the chain of inequalities from expression (23), that

$$
\widetilde{m}(\theta(f), r) \leq 4 \cdot \widetilde{m}(f, r)+(11 n+3) \cdot \log (r)+F
$$

for big $r$ outside a set of finite linear measure. Hence, using the correct constants from expressions (31) and (34) the results is proved.

We are now ready to prove Theorem 4.2.

Proof of Theorem 4.2. By expression (18) we have that

$$
\widetilde{m}(\theta(f), r) \leq \widetilde{m}\left(\theta\left(f_{\text {inv }}\right), r\right)+\widetilde{m}\left(\theta\left(f_{\text {anti }}\right), r\right)+C .
$$

By Lemmas 4.6 and 4.7 we have that the right-hand side of (35) is less than or equal to

$$
3 \cdot \widetilde{m}\left(f_{\text {inv }}, r\right)+4 \cdot \log (r)+4 \cdot \widetilde{m}\left(f_{\text {anti }}, r\right)+E(n) \cdot \log (r)+F
$$

for big $r$ outside a set of finite linear measure. As $f_{\text {inv }}=\frac{f+f \circ I}{2}$ we get

$$
\widetilde{m}\left(f_{\text {inv }}, r\right)=\widetilde{m}\left(\frac{f+f \circ I}{2}, r\right) \leq \widetilde{m}\left(\frac{f}{2}, r\right)+\widetilde{m}\left(\frac{f \circ I}{2}, r\right)+C
$$

by property 1 in Lemma 3.3. Using Lemma 4.4 yields that the right-hand side of inequality (37) equals

$$
2 \cdot \widetilde{m}(f, r)+G
$$

for some constant $G$, so expression (35) together with expressions (36) and (37) yields $\widetilde{m}(\theta(f), r) \leq 3(2 \cdot \widetilde{m}(f, r)+G)+4 \log r+4 \cdot \widetilde{m}\left(f_{\text {anti }}, r\right)+E(n) \cdot \log (r)+F$ 
Using that $f_{\text {anti }}=\frac{f \circ I-f}{2}$ yields, by similar estimates as for $f_{\text {inv }}$, that

$$
\widetilde{m}\left(f_{\text {anti }}, r\right) \leq 2 \cdot \widetilde{m}(f, r)+H
$$

Combining equations (39) and (40) gives

$$
\begin{aligned}
\widetilde{m} & (\theta(f), r) \\
& \leq 3(2 \cdot \widetilde{m}(f, r)+G)+4 \cdot \log (r)+4(2 \cdot \widetilde{m}(f, r)+H)+E(n) \cdot \log (r)+F \\
& =14 \cdot \widetilde{m}(f, r)+K(n)+L
\end{aligned}
$$

for big $r$ outside a set of finite linear measure, where $K(n)$ is an affine polynomial and $L$ is a constant.

The above theorem will be used for estimating the volume change of an overshear. Recall from the work of Kaliman and Kutzschebauch in [9] that the Danielewski surfaces admit a unique up to a nonzero constant algebraic "volume form" $\omega$, in the sense that is in every point of maximal complex rank. In the open dense chart corresponding to local coordinates on $\mathbb{C}_{x}^{*} \times \mathbb{C}_{z} \rightarrow D_{p}$ defined by $(x, z) \mapsto\left(x, \frac{p(z)}{x}, z\right)$, yields that the volume form is given by $\omega=\frac{d x \wedge d z}{x}$ (thus fixing the constant, which is however not important for the calculation of volume change). The volume change of a self map $F: D_{p} \rightarrow D_{p}$ of the Danielewski surface,

$$
F(x, y, z)=(u(x, y, z), v(x, y, z), w(x, y, z))
$$

is due to the local expression of $\omega$ given by

$$
|\operatorname{Jac}(F)|=\frac{x}{u(x, z)}\left(\frac{\partial u}{\partial x} \frac{\partial w}{\partial z}-\frac{\partial u}{\partial z} \frac{\partial w}{\partial x}\right)
$$

in coordinates $(x, z)$ in the above chart. Thus we need to estimate the growth of the derivative $\frac{\partial f}{\partial x}$ and $\frac{\partial f}{\partial z}$ by the growth of $f$ itself. To do so we push forward the vector fields $\frac{\partial}{\partial x}$ and $\frac{\partial}{\partial z}$ from $\mathbb{C}_{x}^{*} \times \mathbb{C}_{z}$ to the Danielewski surface, express them as a linear combination (with meromorphic coefficients) of the fields $\theta_{1}$ and $\theta_{2}$ from equations (16) and (17) and use the corresponding estimates.

The pushed forward fields are the meromorphic vector fields

$$
\theta=\frac{\partial}{\partial x}-\frac{y}{x} \frac{\partial}{\partial y}
$$

and

$$
\tilde{\theta}=\frac{p^{\prime}(z)}{x} \frac{\partial}{\partial y}+\frac{\partial}{\partial z} .
$$


The corresponding linear combinations are

$$
\theta=\frac{x-y}{x} \theta_{1}
$$

and

$$
\tilde{\theta}=\theta_{2}+\frac{p^{\prime}(z)}{x} \theta_{1}
$$

where $\theta_{1}$ and $\theta_{2}$ are the vector fields from expressions (16) and (17). Given a holomorphic function $f: D_{p} \rightarrow \mathbb{C}$ we have

$$
\widetilde{m}(\theta(f), r)=\widetilde{m}\left(\frac{x-y}{x} \theta_{1}(f), r\right) \leq \widetilde{m}\left(1-\frac{y}{x}, r\right)+\widetilde{m}\left(\theta_{1}(f), r\right)
$$

by property 2 in Lemma 3.3. Using Theorem 4.2, properties 2 and 3 in Lemma 3.3, and using Lemma 3.8, yields that the right-hand side of (44) is less than or equal to

$$
\begin{aligned}
& \widetilde{m}(y, r)+\widetilde{m}(x, r)+C+14 \cdot \widetilde{m}(f, r)+K(n) \cdot \log (r)+L \\
& \quad=2 n \cdot \log (r)+C+\widetilde{m}(f, r)+K(n) \cdot \log (r)+L,
\end{aligned}
$$

where $C$ is the constant from property 3 in Lemma 3.3, for big $r$ outside a set of finite linear measure. Putting $\tilde{L}=L+C$ and $\tilde{K}(n)=K(n)+2 n$ we get that

$$
\widetilde{m}(\theta(f), r) \leq 14 \cdot \widetilde{m}(f, r)+\tilde{K}(n) \cdot \log (r)+\tilde{L} .
$$

Similar calculations with $\tilde{\theta}$ instead of $\theta$ yield that

$$
\tilde{m}(\tilde{\theta}(f), r) \leq 28 \cdot \widetilde{m}(f, r)+\tilde{K}(n) \cdot \log (r)+\tilde{L}
$$

Thus we have proved

Proposition 4.8 Given a holomorphic function $f: D_{p} \rightarrow \mathbb{C}$, and a vector field $\theta$ which is the push forward of a partial derivative from the chart $\alpha: \mathbb{C}_{x}^{*} \times \mathbb{C}_{z} \rightarrow D_{p}$ given by $(x, z) \mapsto\left(x, \frac{p(z)}{x}, z\right)$, then we have the estimate

$$
\widetilde{m}(\theta(f), r) \leq A \cdot \widetilde{m}(f, r)+K(n) \cdot \log (r)+L
$$

for big $r$ outside a set of finite linear measure, where $K(n), A$ and L are constants. In other words: If we denote the function $f \circ \alpha$ on the chart again by $f$, then

$$
\tilde{m}\left(\frac{\partial f}{\partial x}, r\right) \leq A \cdot \widetilde{m}(f, r)+K(n) \cdot \log (r)+L
$$


and

$$
\widetilde{m}\left(\frac{\partial f}{\partial z}, r\right) \leq A \cdot \widetilde{m}(f, r)+K(n) \cdot \log (r)+L
$$

for big $r$ outside a set of finite linear measure.

We will also use another coordinate chart on the Danielewski surface. Namely, around a point $\left(0,0, z_{0}\right)$ with $p\left(z_{0}\right)=0$ we can use $x$ and $y$ as coordinates, since $p^{\prime}\left(z_{0}\right) \neq 0$. We do not give an explicit formula, but the chart exists by the inverse function theorem. In such coordinates the volume form $\omega$ is given by $\frac{1}{p^{\prime}(z)} d x \wedge d y$ and to estimate the volume change in this chart we use the following proposition which follows exactly as Proposition 4.8.

Proposition 4.9 Given a holomorphic function $f: D_{p} \rightarrow \mathbb{C}$, and identify $f$ and its derivatives with the corresponding functions on the $(x, y)$-chart then the estimates

$$
\widetilde{m}\left(\frac{\partial f}{\partial x}, r\right) \leq C \cdot \widetilde{m}(f, r)+D(n) \cdot \log (r)+E
$$

and

$$
\widetilde{m}\left(\frac{\partial f}{\partial y}, r\right) \leq C \cdot \widetilde{m}(f, r)+D(n) \cdot \log (r)+E
$$

hold for big $r$ outside a set of finite linear measure and for some constants $C, D(n)$ and $E$.

\section{Application to the Overshear Group}

In [1], Ahern and Rudin showed that the overshear group in $\mathbb{C}^{2}$ is a free amalgamated product of the affine automorphisms and the elementary (or Jonquiere) automorphisms over their intersection. The analogous result for the polynomial automorphism group was shown by van der Kulk [20] and Jung [8]. In [14] the polynomial automorphism was determined in the sense that all its generators were given explicitly. Furthermore, Makar-Limanov showed, analogously to van der Kulk's and Jung's work, that $\operatorname{Aut}_{\text {pol }}\left(D_{p}\right)$ has a structure of an amalgamated product. More precisely, he showed that

Assume that $T_{1}$ is generated by shears $S_{f}$ and that $T_{2}$ is generated by shears $I S_{f} I$, where $f \in \mathbb{C}[x]$ is a polynomial. Also consider $H$, the group generated by $H_{\lambda}(x, y, z)=\left(\lambda x, \lambda^{-1} y, z\right)$, and $I_{2}$ the group generated by the involution $I(x, y, z)=(y, x, z)$. Then

$$
\operatorname{Aut}_{\mathrm{pol}}\left(D_{p}\right)=T_{1} * T_{2} \rtimes\left(H \rtimes I_{2}\right) \cong T_{1} * T_{2} \rtimes\left(\mathbb{C}^{*} \rtimes \mathbb{Z}_{2}\right) .
$$

In the paper [15], Makar-Limanov studied $D_{p}^{n}$ and then he showed that 


$$
\operatorname{Aut}_{\mathrm{pol}}\left(D_{p}^{n}\right)=\mathbb{C}[x] \rtimes \mathbb{C}^{*} .
$$

Both of these results were proved in a more general setting in [14] and [15]. Here we have restricted ourselves to the special case when $p$ has simple zeros only, since we want to consider only manifolds.

Ahern and Rudin used the Nevanlinna characteristic to show a similar structure theorem for the overshear group of $\mathbb{C}^{2}$. Following the outline of their proof we will prove the following theorem.

Theorem 5.1 Let $D_{p}$ be a Danielewski surface and assume that $\operatorname{deg}(p) \geq 4$. Then the overshear group, $O S\left(D_{p}\right)$, is a free amalgamated product of $O_{1}$ and $O_{2}$, where $O_{1}$ is generated by $O_{f, g}$ and $O_{2}$ generated by $I O_{f, g} I$.

Assume throughout this section that $n=\operatorname{deg}(p) \geq 4$.

The starting point of the proof of Theorem 5.1 uses the following lemma:

Lemma 5.2 Every composition of overshear mappings is conjugate to the form

$$
I \circ O_{f_{1}, g_{1}} \circ I \circ O_{f_{2}, g_{2}} \circ I \circ \cdots \circ O_{f_{n}, g_{n}}
$$

or to $I$.

Proof We can assume that we have one of the following composition of overshear mappings:

$$
\begin{aligned}
& O_{f_{1}, g_{1}} \circ I \circ O_{f_{2}, g_{2}} \circ I \circ \cdots \circ I \circ O_{f_{n-1}, g_{n-1}} \circ I \circ O_{f_{n}, g_{n}}, \\
& O_{f_{1}, g_{1}} \circ I \circ O_{f_{2}, g_{2}} \circ \cdots \circ I \circ O_{f_{n}, g_{n}} \circ I, \\
& I \circ O_{f_{1}, g_{1}} \circ I \circ O_{f_{2}, g_{2}} \circ \cdots \circ I \circ O_{f_{n-1}, g_{n-1}} \circ I \circ O_{f_{n}, g_{n}}, \\
& I \circ O_{f_{1}, g_{1}} \circ I \circ O_{f_{2}, g_{2}} \circ \cdots \circ I \circ O_{f_{n}, g_{n}} \circ I .
\end{aligned}
$$

We handle each of these compositions separately. We start with expression (48). If we first conjugate with $O_{f_{1}, g_{1}}$ and then with $I$ we obtain

$$
O_{f_{2}, g_{2}} \circ I \circ \cdots \circ I \circ O_{f_{n-1}, g_{n-1}} \circ I \circ O_{f_{n}+f_{1}, g_{1} \cdot e^{x f_{n}}+g_{n}} \circ I .
$$

This is the desired form, but if $e^{f_{n}+f_{1}} \equiv 1$ and $g_{1} \cdot e^{x f_{n}}+g_{n} \equiv 0$, then

$$
O_{f_{n}+f_{1}, g_{1} \cdot e^{x f_{n}}+g_{n}}=\mathrm{id},
$$

and we are back where we started, i.e.,

$$
O_{f_{2}, g_{2}} \circ I \circ \cdots \circ I \circ O_{f_{n-1}, g_{n-1}} .
$$

The worst-case scenario is that after a finite number of conjugations with $O_{f_{k}, g_{k}}$ and $I$ we end up with

$$
O_{f_{j}, g_{j}} \circ I \circ O_{f_{j+1}, g_{j+1}},
$$


for some $j$. If we conjugate one last time we get

$$
O_{f_{j+1}+f_{j}, g_{j} \cdot e^{x f_{j+1}+g_{j+1}}} \circ I .
$$

This gives the conclusion of the lemma even if $e^{f_{j+1}+f_{j}} \equiv 1$ and $g_{j} \cdot e^{x f_{j+1}}+g_{j+1} \equiv 0$.

Expression (49) is already of the desired form, so let us consider expression (50) instead. In this equation it suffices to conjugate with $I$. Finally, consider expression (51). If we start to conjugate with $I$, then we get a sequence as in expression (48), and this case was handled above.

Look at an overshear mapping composed with an involution mapping on the Danielewski surface $D_{p}$

$$
\left(u_{1}, v_{1}, w_{1}\right)=\left(\frac{p\left(z f_{1}(x)+x g_{1}(x)\right)}{x}, x, z f_{1}(x)+x g_{1}(x)\right)
$$

and at a finite composition of such mappings

$$
\begin{aligned}
& \left(u_{k+1}, v_{k+1}, w_{k+1}\right) \\
& \quad=\left(\frac{p\left(w_{k} f_{k+1}\left(u_{k}\right)+u_{k} g_{k+1}\left(u_{k}\right)\right)}{u_{k}}, u_{k}, w_{k} f_{k+1}\left(u_{k}\right)+u_{k} g_{k+1}\left(u_{k}\right)\right),
\end{aligned}
$$

where $k \geq 1, f_{j}: \mathbb{C} \rightarrow \mathbb{C}^{*}$ is holomorphic and $f_{j}(0)=1$ (thus either constantly 1 or transcendental), and $g_{j}: \mathbb{C} \rightarrow \mathbb{C}$ is holomorphic.

Consider the mapping in expression (52). Since $u_{1} \cdot v_{1}=p\left(w_{1}\right)$ on $D_{p}$, we have that $u_{1}=\frac{p\left(w_{1}\right)}{v_{1}}=\frac{p\left(w_{1}\right)}{x}$. By expression (41) we get that the determinant of the Jacobian of $\left(u_{1}, v_{1}, w_{1}\right)$ equals

$$
\frac{x^{2}}{p\left(w_{1}\right)}\left(\frac{\partial u_{1}}{\partial x} \frac{\partial w_{1}}{\partial z}-\frac{\partial u_{1}}{\partial z} \frac{\partial w_{1}}{\partial x}\right) .
$$

Since $u_{1}=\frac{p\left(w_{1}\right)}{x}$, then

$$
\frac{\partial u_{1}}{\partial x}=\frac{p^{\prime}\left(w_{1}\right) \frac{\partial w_{1}}{\partial x} x-p\left(w_{1}\right)}{x^{2}}
$$

and

$$
\frac{\partial u_{1}}{\partial z}=\frac{p^{\prime}\left(w_{1}\right) \frac{\partial w_{1}}{\partial z}}{x}
$$

Inserting expressions (55) and (56) in (54) yields that

$$
\frac{x^{2}}{p\left(w_{1}\right)}\left(\frac{p^{\prime}\left(w_{1}\right) \frac{\partial w_{1}}{\partial x} \frac{\partial w_{1}}{\partial z} x-p\left(w_{1}\right) \frac{\partial w_{1}}{\partial z}}{x^{2}}-\frac{p^{\prime}\left(w_{1}\right) \frac{\partial w_{1}}{\partial z} \frac{\partial w_{1}}{\partial x}}{x}\right)=-\frac{\partial w_{1}}{\partial z}
$$


Thus the chain rule implies that

Lemma 5.3 The following relation holds:

$$
\frac{\left|\operatorname{Jac}\left(u_{k+1}, v_{k+1}, w_{k+1}\right)\right|}{\left|\operatorname{Jac}\left(u_{k}, v_{k}, w_{k}\right)\right|}=-f_{k+1}\left(u_{k}\right)
$$

An important step in the proof of Theorem 5.1 are the following lemmas. The proofs of our two key lemmas use estimates of the determinant of the Jacobian.

Lemma 5.4 The functions from expressions (52) and (53) fulfill

$$
\widetilde{m}\left(\left|\operatorname{Jac}\left(u_{k}, v_{k}, w_{k}\right)\right|, r\right) \leq A \cdot \widetilde{m}\left(u_{k}, r\right)+B \cdot \widetilde{m}\left(v_{k}, r\right)+C \cdot \log (r)+D,
$$

for $k \geq 1$, for certain constants $A, B, C, D$, for big $r$ outside a set of finite linear measure.

Proof As

$$
\left|\operatorname{Jac}\left(u_{k}, v_{k}, w_{k}\right)\right|=\frac{p^{\prime}(z)}{p^{\prime}\left(w_{k}\right)}\left(\frac{\partial u_{k}}{\partial x} \frac{\partial v_{k}}{\partial y}-\frac{\partial u_{k}}{\partial y} \frac{\partial v_{k}}{\partial x}\right)
$$

we get the following estimate by properties 1 and 2 in Lemma 3.3

$$
\begin{aligned}
\widetilde{m}\left(\left|\operatorname{Jac}\left(u_{k}, v_{k}, w_{k}\right)\right|, r\right) \leq & \widetilde{m}\left(\frac{p^{\prime}(z)}{p^{\prime}\left(w_{k}\right)}, r\right)+\widetilde{m}\left(\frac{\partial u_{k}}{\partial x}, r\right) \\
& +\widetilde{m}\left(\frac{\partial v_{k}}{\partial y}, r\right)+\widetilde{m}\left(\frac{\partial u_{k}}{\partial y}, r\right)+\widetilde{m}\left(\frac{\partial v_{k}}{\partial x}, r\right)+\log 2
\end{aligned}
$$

By property 3 in Lemma 3.3 and by Proposition 4.9 we get that the right-hand side of (58) is less than or equal to

$$
\begin{aligned}
& \widetilde{m}\left(p^{\prime}(z), r\right)+\widetilde{m}\left(p^{\prime}\left(w_{k}\right), r\right) \\
& \quad+2 C \cdot \widetilde{m}\left(u_{k}, r\right)+2 C \cdot \widetilde{m}\left(v_{k}, r\right)+4 D \cdot \log (r)+\tilde{E},
\end{aligned}
$$

where $\tilde{E}$ is the constant $4 E+\log 2$, for big $r$ outside a set of finite linear measure. Using Corollary 3.6 yields that the right-hand side of (59) equals

$$
\begin{aligned}
& (n-1) \cdot \widetilde{m}(z, r)+(n-1) \cdot \widetilde{m}\left(w_{k}, r\right) \\
& \quad+2 C \cdot \widetilde{m}\left(u_{k}, r\right)+2 C \cdot \widetilde{m}\left(v_{k}, r\right)+4 D \cdot \log (r)+\tilde{E}_{2},
\end{aligned}
$$

where $\tilde{E}_{2}$ is a constant. By Lemma 3.8 we get that expression (60) tends to

$$
\begin{aligned}
& (n-1) 2 \log (r)+(n-1) \cdot \widetilde{m}\left(w_{k}, r\right) \\
& \quad+2 C \cdot \widetilde{m}\left(u_{k}, r\right)+2 C \cdot \widetilde{m}\left(v_{k}, r\right)+4 D \cdot \log (r)+\tilde{E}_{2}
\end{aligned}
$$


for big $r$ outside a set of finite linear measure. Using that $u_{k} v_{k}=p\left(w_{k}\right)$ and using property 2 in Lemma 3.3 and Corollary 3.6 we get that

$$
\widetilde{m}\left(u_{k} v_{k}, r\right)=\widetilde{m}\left(p\left(w_{k}\right), r\right)=n \cdot \widetilde{m}\left(w_{k}, r\right)+F
$$

which implies

$$
\widetilde{m}\left(w_{k}, r\right) \leq \frac{\widetilde{m}\left(u_{k}, r\right)+\widetilde{m}\left(v_{k}, r\right)-F}{n} .
$$

Combining the chain of inequalities from expression (58) to expression (61) with expression (63) yields

$$
\widetilde{m}\left(\left|\operatorname{Jac}\left(u_{k}, v_{k}, w_{k}\right)\right|, r\right) \leq A \cdot \widetilde{m}\left(u_{k}, r\right)+B \cdot \widetilde{m}\left(v_{k}, r\right)+C \cdot \log (r)+D
$$

for big $r$ outside a set of finite linear measure and properly chosen constants $A, B, C, D$.

Lemma 5.5 The functions $u_{1}$ and $v_{1}$ in equation (52) fulfill the estimate

$$
\frac{\widetilde{m}\left(u_{1}, r\right)}{\widetilde{m}\left(v_{1}, r\right)} \geq 2
$$

for big $r$ outside a set of finite linear measure, provided $\operatorname{deg} p=n \geq 3$.

Proof We will consider two cases.

Case 1: $f_{1}$ is identically 1 .

We have $u_{1}(x, z)=\frac{p\left(z+x g_{1}(x)\right)}{x}$ and $v_{1}(x, z)=x$. Therefore,

$$
\frac{\widetilde{m}\left(\frac{p\left(z+x g_{1}(x)\right)}{x}, r\right)}{\widetilde{m}(x, r)} \geq \frac{\widetilde{m}\left(p\left(z+x g_{1}(x)\right), r\right)-\widetilde{m}(x, r)}{\widetilde{m}(x, r)}
$$

by property 2 in Lemma 3.3. If $g$ is transcendental then Corollary 3.6, property 1 in Lemma 3.3 together with Proposition 3.7 yields that

$$
\begin{aligned}
\lim _{r \rightarrow \infty} \frac{\tilde{m}\left(u_{1}, r\right)}{\widetilde{m}\left(v_{1}, r\right)} & \geq \lim _{r \rightarrow \infty} \frac{n \cdot \tilde{m}\left(x g_{1}(x), r\right)-n \cdot \tilde{m}(z, r)-\tilde{m}(x, r)-n D}{\tilde{m}(x, r)} \\
& =\infty-\frac{2 n}{n}-1-0=\infty
\end{aligned}
$$

Assume now that $g_{1}$ is a polynomial. Then $u_{1}$ is a polynomial in $x, y$ and $z$ with highest order term $x^{n-1} \cdot\left(g_{1}(x)\right)^{n}$. From Lemma 3.8 and Corollary 3.6 it follows that

$$
\lim _{r \rightarrow \infty} \frac{\widetilde{m}\left(u_{1}, r\right)}{\widetilde{m}\left(v_{1}, r\right)} \geq(n-1)+n \cdot \operatorname{deg}\left(g_{1}\right) \geq n-1 \geq 2
$$


and the first case is finished.

Case 2: $f_{1}$ is transcendental.

Combining expressions (52) and (57) we see that

$$
\left|\operatorname{Jac}\left(u_{1}, v_{1}, w_{1}\right)\right|=-f(x)
$$

Therefore, Lemma 5.4 yields

$$
\widetilde{m}(-f(x), r) \leq A \cdot \widetilde{m}\left(u_{1}, r\right)+B \cdot \widetilde{m}\left(v_{1}, r\right)+C \cdot \log (r)+D,
$$

for constants $A, B, C$ and $D$. Dividing with $\widetilde{m}\left(v_{1}, r\right)$ and $A$ in expression (66) yields

$$
\frac{\widetilde{m}\left(u_{1}, r\right)}{\widetilde{m}\left(v_{1}, r\right)} \geq \frac{1}{A} \frac{\widetilde{m}(-f(x), r)}{\widetilde{m}(x, r)}-\frac{B}{A}-\frac{C \log r+D}{A \cdot \widetilde{m}(x, r)}=\infty-\frac{B}{A}-0=\infty
$$

for big $r$ outside a set of finite linear measure, by Proposition 3.7.

Lemma 5.6 Let $u, v, w: D_{p} \rightarrow \mathbb{C}$ be holomorphic functions defined on the Danielewski surface with $\operatorname{deg} p=n$, satisfying uv $=p(w)$. Let $f: \mathbb{C} \rightarrow \mathbb{C}^{*}$ and $g: \mathbb{C} \rightarrow \mathbb{C}$ be holomorphic, with $f(0)=1$. Assume that

$$
\begin{gathered}
\frac{\widetilde{m}(u, r)}{\widetilde{m}(v, r)}>1+\delta \text { for some } \delta \geq 0 \\
\quad \text { and } \\
n \geq 5 \text { or }(n=4 \text { and } \delta>0)
\end{gathered}
$$

for big $r$ outside a set of finite linear measure. Then the functions $U, V$ defined by

$$
(U, V, W)=\left(\frac{p(w f(u)+u g(u))}{u}, u, w f(u)+u g(u)\right)
$$

fulfill

$$
\frac{\widetilde{m}(U, r)}{\widetilde{m}(V, r)}>1+\varepsilon
$$

for big $r$ outside a set of finite linear measure too, where

$$
\varepsilon=\frac{1}{2} \frac{\delta}{1+\delta}
$$

In fact, $\varepsilon$ can be chosen arbitrarily large if $f$ or $g$ is transcendental, even if $\delta=0$. 
Proof We start with an observation: The relation $u \cdot v=p(w)$ implies, together with Lemma 3.8 and Proposition 3.4 that

$$
\widetilde{m}(w, r) \leq \frac{\widetilde{m}(u, r)+\widetilde{m}(v, r)-C}{n}, \quad C \in \mathbb{R}
$$

which results in

$$
\frac{\widetilde{m}(w, r)}{\widetilde{m}(u, r)} \leq\left(\frac{1}{n} \frac{\widetilde{m}(v, r)}{\widetilde{m}(u, r)}+\frac{1}{n}-\frac{C}{n \cdot \widetilde{m}(u, r)}\right)<\frac{1}{n} \cdot\left(2-\frac{\delta}{1+\delta}\right),
$$

for big $r$ outside a set of finite linear measure by the hypothesis in the lemma. We will now consider two different cases as we did in Lemma 5.5.

Case 1: $f$ is identically 1 .

$$
\begin{aligned}
\frac{\widetilde{m}(U, r)}{\widetilde{m}(V, r)}=\frac{\widetilde{m}\left(\frac{p(w+u \cdot g(u))}{u}, r\right)}{\widetilde{m}(u, r)} & \geq \frac{\widetilde{m}(p(w+u \cdot g(u)), r)-\widetilde{m}(u, r)}{\widetilde{m}(u, r)} \\
& \geq \frac{n \cdot \widetilde{m}(u \cdot g(u), r)-n \cdot \widetilde{m}(w, r)-\widetilde{m}(u, r)+C}{\widetilde{m}(u, r)} \\
& \geq n \cdot \frac{\widetilde{m}(u \cdot g(u), r)}{\widetilde{m}(u, r)}-3+\frac{\delta}{1+\delta}+O\left(\frac{1}{\log (r)}\right)
\end{aligned}
$$

(a) If $g$ is transcendental, then the first term in the sum tends to infinity, $\varepsilon$ can be chosen arbitrarily large.

(b) If $g$ is a polynomial (in the worst case, $g$ is constant), then we need to have that $n-3+\frac{\delta}{1+\delta}>1+\varepsilon$. This can be satisfied with $\varepsilon=\frac{1}{2} \frac{\delta}{1+\delta}$ if $n \geq 5$ or $n=4$ and $\delta>0$.

Case 2: $f$ is transcendental

Lemmas 5.3, 5.4 and Properties 2 and 3 in Lemma 3.3 yield

$$
\begin{aligned}
\widetilde{m}(-f(u), r)= & \widetilde{m}\left(\frac{|\operatorname{Jac}(U, V, W)|}{|\operatorname{Jac}(u, v, w)|}, r\right) \\
\leq & A \cdot \widetilde{m}(U, r)+B \cdot \widetilde{m}(V, r)+C \cdot \widetilde{m}(u, r) \\
& +D \cdot \widetilde{m}(v, r)+E \cdot \log (r)+F .
\end{aligned}
$$

Divide expression (69) by $A \cdot \widetilde{m}(V, r)$. Then we get

$$
\frac{\widetilde{m}(U, r)}{\widetilde{m}(V, r)} \geq \frac{1}{A} \frac{\widetilde{m}(-f(u), r)}{\widetilde{m}(V, r)}-\frac{B}{A}-\frac{C \cdot \widetilde{m}(u, r)}{A \cdot \widetilde{m}(V, r)}-\frac{E \cdot \log (r)+F}{A \cdot \widetilde{m}(V, r)}
$$

for big $r$ outside a set of finite linear measure. As $\widetilde{m}(V, r)=\widetilde{m}(u, r)$, we get, by the hypothesis and Proposition 3.7, that the right-hand side of (70) is greater than

$$
\infty-\frac{B}{A}-\frac{C}{A}-0=\infty
$$


In this case we can choose $\varepsilon$ arbitrarily large. Hence, the proof is finished.

We can interpret this lemma in the following way: In case of degree $n \geq 5$, the property $\frac{\widetilde{m}(u, r)}{\widetilde{m}(v, r)}>1$ is preserved under composition with an overshear map. In case of degree $n=4$ this is only true if the fraction happens to be bounded away from 1 .

Proof of Theorem 5.1. We will show that id is not a reduced product. This will show that we cannot get a non-trivial kernel of the mapping

$$
\varphi: O_{1} * O_{2} \rightarrow \operatorname{OS}\left(D_{p}\right)
$$

defined in the obvious way. The mapping $\varphi$ is surjective by construction. To get a contradiction, we assume that id is a reduced product. By Lemma 5.2 we may assume that

$$
\mathrm{id}=I \circ O_{f_{1}, g_{1}} \circ I \circ O_{f_{2}, g_{2}} \circ I \circ \cdots \circ I \circ O_{f_{n}, g_{n}}=: G_{1} \circ \cdots \circ G_{n},
$$

where $G_{i}=I \circ O_{f_{i}, g_{i}}$, for each $i \geq 1$. For $k \leq n$, define functions $u_{k}, v_{k}$ and $w_{k}$ on $D_{p}$ by

$$
\left(u_{k}(x, y, z), v_{k}(x, y, z), w_{k}(x, y, z)\right)=G_{1} \circ \cdots \circ G_{k}(x, y, z) .
$$

The definition of $u_{k}, v_{k}$ and $w_{k}$ are easily verified to be the following functions:

$$
\left(u_{1}, v_{1}, w_{1}\right)=\left(\frac{p\left(z f_{1}(x)+x g_{1}(x)\right)}{x}, x, z f_{1}(x)+x g_{1}(x)\right)
$$

and

$$
\begin{aligned}
& \left(u_{k+1}, v_{k+1}, w_{k+1}\right) \\
& \quad=\left(\frac{p\left(w_{k} f_{k+1}\left(u_{k}\right)+u_{k} g_{k+1}\left(u_{k}\right)\right)}{u_{k}}, u_{k}, w_{k} f_{k+1}\left(u_{k}\right)+u_{k} g_{k+1}\left(u_{k}\right)\right) .
\end{aligned}
$$

These functions are the same as in expressions (52) and (53). Now Lemma 5.5 implies $\frac{\widetilde{m}\left(u_{1}, r\right)}{\widetilde{m}\left(v_{1}, r\right)}>1+\delta_{1}, \delta_{1}>0$, for big $r$ outside a set of finite linear measure. By induction using Lemma 5.6 it follows that $\frac{\widetilde{m}\left(u_{k}, r\right)}{\widetilde{m}\left(v_{k}, r\right)}>1+\delta_{k}, \delta_{k}>0$, for all $k$, for big $r$ outside a set of finite linear measure. As $\lim _{r \rightarrow \infty} \frac{\widetilde{m}(x, r)}{\widetilde{m}(y, r)}=1$, by Lemma 3.8, we get a contradiction. Therefore, $\varphi$ is injective, and by construction also surjective. Hence $\mathrm{OS}\left(D_{p}\right)=O_{1} * O_{2}$.

In general it is known that the overshear group on $\mathbb{C}^{n}$ is a proper subgroup of the holomorphic automorphism group by an argument using the Baire Category theorem due to Andersén and Lempert [3]. However, only for $\mathbb{C}^{2}$ there is a known explicit example of a holomorphic automorphism not belonging to the overshear group; see [2]. For the Danielewski surface we are also able to give such a concrete example. 
Corollary 5.7 Let $p \geq 4$. The automorphism $(x, y, z) \mapsto\left(x e^{z}, y e^{-z}, z\right)$ is not contained in the overshear group, thus $O S\left(D_{p}\right)$ is a proper subgroup of the holomorphic automorphism group of $D_{p}$.

Proof We apply the same proof as for Theorem 5.1. It is sufficient to show that $\widetilde{m}\left(x e^{z}, r\right)=\widetilde{m}\left(y e^{-z}, r\right)$ which by the previous discussion makes it impossible to write this map as a composition of overshears. By symmetry in $x$ and $y$, clearly $\widetilde{m}\left(x e^{z}, r\right)=\widetilde{m}\left(y e^{z}, r\right)$.

$$
\begin{aligned}
\widetilde{m}\left(y e^{-z}, r\right) & =m\left(1 /\left(y e^{-z}\right), r\right)=\int_{\pi^{-1}\left(r S^{3}\right)} \log ^{+}\left|y e^{-z}\right|^{2} \pi^{*} \mathrm{~d} \eta \\
& =\int^{=}-z \int_{\pi^{-1}\left(r S^{3}\right)} \log ^{+}\left|y e^{z}\right|^{2} \pi^{*} \mathrm{~d} \eta \\
& m\left(1 /\left(y e^{z}\right), r\right)=\widetilde{m}\left(y e^{z}, r\right)
\end{aligned}
$$

The last equality holds since $r S^{3}$ is invariant under this coordinate transformation and $\pi(x, y, z)=(x+y, z)$.

Remark 5.8 Let $S_{1}$ be the subgroup of the shear group generated by $S_{f}$ and let $S_{2}$ be generated by $I \circ S_{f} \circ I$. Then it follows from Theorem 5.1 that $\mathrm{S}\left(D_{p}\right)$ is a free product of $S_{1}$ and $S_{2}$ for $\operatorname{deg}(p) \geq 4$. For $\operatorname{deg}(p)=1$ the Danielewski surface is just $\mathbb{C}^{2}$. In this case the conclusion of Theorem 5.1 does not hold. Indeed, the identity, viewed as a $2 \times 2$ matrix, can be written as the product

$$
\left[\begin{array}{cc}
1 & -1 \\
0 & 1
\end{array}\right]\left[\begin{array}{ll}
1 & 0 \\
1 & 1
\end{array}\right]\left[\begin{array}{cc}
1 & -1 \\
0 & 1
\end{array}\right]\left[\begin{array}{cc}
1 & 0 \\
-1 & 1
\end{array}\right]\left[\begin{array}{ll}
1 & 1 \\
0 & 1
\end{array}\right]\left[\begin{array}{cc}
1 & 0 \\
-1 & 1
\end{array}\right]
$$

of shears. If $\operatorname{deg}(p)=3$ the proof of Theorem 5.1 does not work, but the authors do not know whether the theorem holds or not. However, for $\operatorname{deg}(p)=2$ there is the following counterexample:

Example 5.9 (Counterexample for $n=2$ ) Let $p(z)=z^{2}-1=(z+1)(z-1)$ and consider the following map

$$
A: \mathbb{C}^{3} \rightarrow \mathbb{C}^{3},(x, y, z) \mapsto(u, v, w):=(-x+y+2 i z, x, i x+z)
$$

This map induces by restriction a map $A \mid D_{p}: D_{p} \rightarrow D_{p}$, as one easily checks:

$$
u v-p(w)=(y-x+2 i z) \cdot x-(z+i x)^{2}+1=0, \quad \text { using } x y-z^{2}+1=0
$$

By looking at the eigenvalues of the complex linear map $A$, one sees that $A^{6}=\mathrm{id}_{\mathbb{C}^{3}}$. Therefore, the structure theorem cannot hold in this case. 
Acknowledgments The second author was partially supported by Schweizerische Nationalfonds Grants No. 200020-134876/1 and 200021-140235/1.

\section{References}

1. Ahern, P., Rudin, W.: Periodic automorphisms of $\mathbb{C}^{n}$. Indiana Univ. Math. J. 44(2), 173-208 (1985)

2. Andersén, E.: Volume-preserving automorphisms of $\mathbb{C}^{n}$. Complex Var. Theory Appl. 14(1-4), 223-235 (1990)

3. Andersén, E., Lempert, L.: On the group of holomorphic automorphisms of $\mathbb{C}^{n}$. Invent. Math. 110(2), 371-388 (1992)

4. Derksen, H., Kutzschebauch, F.: Nonlinearizable holomorphic group actions. Math. Ann. 311(1), 4153 (1998)

5. Derksen, H., Kutzschebauch, F.: Global holomorphic linearization of actions of compact Lie groups on $\mathbb{C}^{n}$. Contemp. Math. 222, 201-210 (1999)

6. Griffiths, P., King, J.: Nevanlinna theory and holomorphic mappings between algebraic varieties. Acta math. 130, 145-220 (1973)

7. Heinzner, P., Kutzschebauch, F.: An equivariant version of Grauert's Oka principle. Invent. Math. 119(2), 317-346 (1995)

8. Jung, H.W.E.: Über ganze birationale Transformationen der Ebene. J. Reine Angew. Math. 184, 161174 (1942)

9. Kaliman, S., Kutzschebauch, F.: Density property for hypersurfaces $U V=P(\bar{X})$. Math. Z. 258(1), 115-131 (2008)

10. Kraft, H., Kutzschebauch, F.: Affine line bundles and linearization. Math. Res. Lett. 3, 619-627 (1996)

11. Kutzschebauch, F., Lind, A.: Holomorphic automorphisms of Danielewski surfaces I: density of the group of overshears. In: Proceedings of the AMS

12. Kosiński, Ł.: A local form of automorphisms of the spectral unit ball. arXiv:1202.5793

13. Laine, I.: Nevanlinna Theory and Complex Differential Equations. de Gruyter Studies in Mathematics, vol. 15. Walter de Gruyter \& Co., Berlin (1993)

14. Makar-Limanov, L.: On groups of automorphisms of a class of surfaces. Isr. J. Math. 69(2), 250-256 (1990)

15. Makar-Limanov, L.: On the group of automorphisms of a surface $x^{n} y=P(z)$. Isr. J. Math. 121, 113-123 (2001)

16. Makar-Limanov L.: Locally nilpotent derivations on the surface $x y=p(z)$. In: Proceedings of the Third International Algebra Conference (Tainan, 2002), pp. 215-219. Kluwer Acad. Publ., Dordrecht (2003)

17. Makar-Limanov, L.: On the hypersurface $x+x^{2} y+z^{2}+t^{3}=0$ in $\mathbb{C}^{4}$ or a $\mathbb{C}^{3}$-like threefold which is not $\mathbb{C}^{3}$. Isr. J. Math. 96(part B), 419-429 (1996)

18. Mohon'ko, A.Z.: Nevanlinna characteristics of certain meromorphic functions. Teor. Funkc. Funkc. Anal. i Priložen. 14, 83-87 (Russian) (1971)

19. Rosay, J.-P., Rudin, W.: Holomorphic maps from $C^{n}$ to $C^{n}$. Trans. AMS 310(1), $405-417$ (1988)

20. Van der Kulk, W.: On polynomial rings in two variables. Nieuw Arch. Wiskd. 1(3), 33-41 (1953)

21. Varolin, D.: The density property for complex manifolds and geometric structures. J. Geom. Anal. 11, 135-160 (2001)

22. Varolin, D.: The density property for complex manifolds and geometric structures II. Int. J. Math. 11(6), 837-847 (2000) 